\title{
A patient with encephalitis associated with NMDA receptor antibodies
}

\author{
Lauren H Sansing, Erdem Tüzün, Melissa W Ko, Jennifer Baccon, David R Lynch and Josep Dalmau*
}

\section{SUMMARY}

Background A 34-year-old woman presented with headache, feverish sensation and anxiety, rapidly followed by homicidal ideation, aggressive agitation, seizures, hypoventilation, hyperthermia and prominent autonomic instability requiring intubation and sedation. She developed episodes of hypotension and bradycardia with periods of asystole lasting up to 15 seconds. Upon weaning off sedation, her eyes opened but she was unresponsive to stimuli. There was muscle rigidity, frequent facial grimacing, rhythmic abdominal contractions, kicking motions of the legs, and intermittent dystonic postures of the right arm.

Investigations Routine laboratory testing, toxicology screening, studies for autoimmune and infectious etiologies, brain MRI scan, lumbar puncture, electroencephalogram, whole-body CT scan, abdominal ultrasound, paraneoplastic and voltage-gated potassium channel antibody serologies, analysis of $\mathrm{N}$-methyl-D-aspartate receptor antibodies.

Diagnosis Paraneoplastic encephalitis associated with immature teratoma of the ovary and $N$-methyl-D-aspartate receptor antibodies.

Management Intensive care, mechanical ventilation, antiepileptics, laparotomy and left salpingo-oophorectomy, corticosteroids, plasma exchange, intravenous immunoglobulin, cyclophosphamide, physical therapy, and chemotherapy.

KEYWORDS catatonia, NMDA receptor antibodies, ovarian teratoma, paraneoplastic encephalitis, psychosis

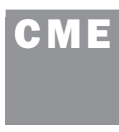

LH Sansing is a Stroke Fellow, MW Ko is a Resident in Neurology, and J Baccon is a Neuropathology Fellow, all at the Hospital of the University of Pennsylvania. E Tüzün is a Senior Research Investigator at the Laboratory of Neuro-oncology and Paraneoplastic Disorders, DR Lynch is Associate Professor of Neurology and Pediatrics, and J Dalmau is Professor of Neurology (Division Neuro-oncology) and Director of the Laboratory of Neurooncology and Paraneoplastic Disorders, all at the University of Pennsylvania, Philadelphia, PA, USA.

\section{Correspondence}

*Department of Neurology, 3 West Gates (Division of Neuro-oncology), University of Pennsylvania, 3400 Spruce Street, Philadelphia, PA 19104, USA

josep.dalmau@uphs.upenn.edu
Vanderbilt Continuing Medical Education online This article offers the opportunity to earn one Category 1 credit toward the AMA Physician's Recognition Award.

\section{THE CASE}

For 1 week, a 34-year-old woman complained of headache, feeling feverish, and being unsure of herself. She attributed these symptoms to stress and anxiety and took two of her husband's alprazolam pills one afternoon. The following day, she was found confused and was brought to the emergency room. Upon arrival she had generalized convulsions that were treated with lorazepam $4 \mathrm{mg}$ intravenously and phenytoin $1,000 \mathrm{mg}$ intravenously, and she was intubated for airway protection. Her temperature was $38.7^{\circ} \mathrm{C}$; other vital signs, general examination, routine blood studies, and urine toxicology screening were normal. A head CT scan was unremarkable; cerebrospinal fluid (CSF) analysis showed a white blood cell count of 18 cells/ml (98\% lymphocytes), red blood cell count of 26 cells $/ \mathrm{ml}$, glucose $4.27 \mathrm{mmol} / \mathrm{l}(77 \mathrm{mg} / \mathrm{dl})$, and protein $0.55 \mathrm{~g} / \mathrm{l}$. Aciclovir $10 \mathrm{mg} / \mathrm{kg}$ body weight every 8 hours was started for possible herpes simplex virus (HSV) encephalitis. MRI fluid-attenuated inversion recovery (FLAIR) showed bilateral medial temporal lobe hyperintensity, predominantly involving the left hippocampus (Figure 1A). An electroencephalogram (EEG) showed 8-12 Hz mixed polymorphic alpha activity, without focal slowing, spikes or sharp waves. HSV polymerase chain reaction (PCR) was negative and aciclovir was discontinued. The patient's mental status improved, and she was discharged home on levetiracetam $500 \mathrm{mg}$ twice daily.

The following evening the patient awoke after having visions that she would stab and kill her 3-year-old son, and asked to be taken back to the hospital. On arrival, vital signs and neurologic examination were normal. She was tearful with pressured speech, and denied hearing voices or having suicidal ideations. She was diagnosed with acute psychosis and readmitted. 

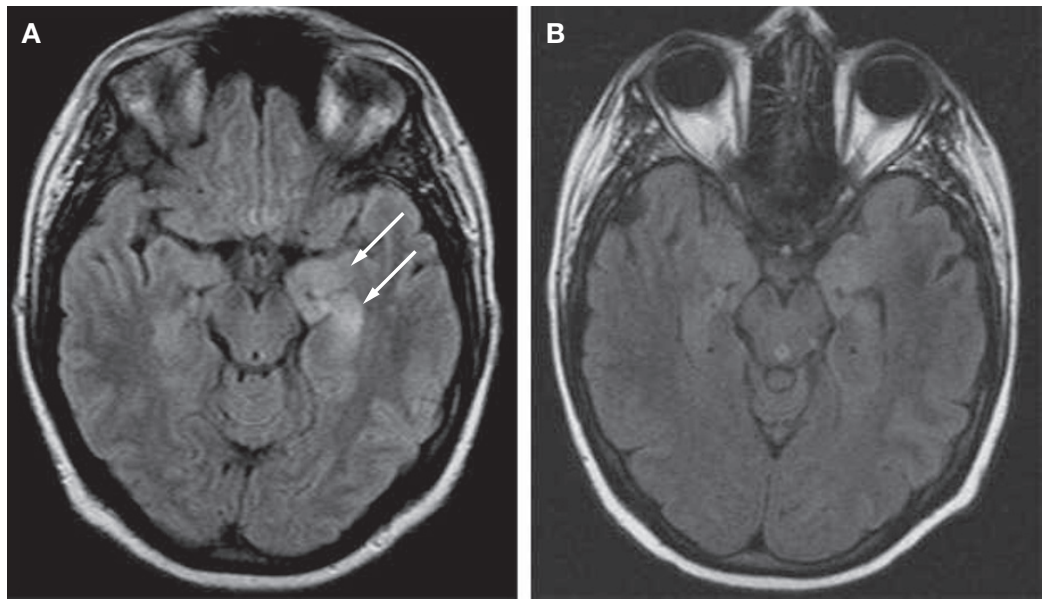

Figure $1 \mathrm{MRI}$ scan of the patient at symptom presentation and follow-up. (A) MRI fluid-attenuated inversion recovery (FLAIR) obtained at symptom presentation demonstrates bilateral medial temporal lobe hyperintense signal, predominantly involving the left hippocampus (arrows). (B) Follow-up MRI obtained during recovery, 4 months after the initial MRI, shows considerable improvement of the FLAIR hyperintensity.
The patient's medical history was notable for obesity and hyperglycemia, both attributed to a polycystic ovarian syndrome. She did not smoke, drink alcohol, or use illegal drugs. One sister had scleroderma and another had systemic lupus erythematosus.

Levetiracetam was discontinued and valproic acid loaded at $1,500 \mathrm{mg}$ intravenously and then continued at $500 \mathrm{mg}$ three times daily. CSF analyses were similar to those previously obtained; bacterial and fungal studies, cytology and flow cytometry were unrevealing. Tests for Lyme disease, Epstein-Barr virus, and arboviruses were negative. Aciclovir was restarted at the same dosage as previously, although a repeat HSV PCR was negative. The patient was given lorazepam $1-2 \mathrm{mg}$ intravenously every 2 hours as needed and olanzapine $5 \mathrm{mg}$ daily for aggressive agitation. Over the next few days she became less communicative, stopped following commands, and developed catatonic features. MRI showed persistent FLAIR hyperintensity in the hippocampi and mild increased meningeal enhancement. An EEG demonstrated $2-6 \mathrm{~Hz}$ polymorphic delta and theta activity, without epileptic activity. She developed episodes of hypoventilation, hypotension (around $80 / 30 \mathrm{mmHg}$ ), and bradycardia (30 beats per minute) with periods of asystole lasting up to 15 seconds. A transthoracic echocardiogram was normal, and the patient was transferred to the neurointensive care unit of another institution.
On arrival, the patient's temperature was $39.4^{\circ} \mathrm{C}$; she was intubated and sedated. Her sedation was immediately discontinued. Several hours later, she remained unresponsive; her eyes would open without tracking or blinking to threat. The patient's pupils were reactive and her oculocephalic and gag reflexes were intact. Muscle tone was increased, with rigidity and normal reflexes. She had frequent facial grimacing, rhythmic abdominal contractions, kicking motions of the legs, and intermittent dystonic postures of the right arm. An EEG showed no epileptiform correlate to the motor activity, but because of concern for an occult epileptic focus, topiramate $100 \mathrm{mg}$ twice daily was added. Testing for HIV, rapid plasma reagin, and a comprehensive panel for autoimmunity (including among others anti-double-stranded DNA, thyroid peroxidase, and antineutrophil cytoplasmic antibodies) were negative. CSF analysis showed a white blood cell count of 30 cells $/ \mathrm{ml}$ ( $97 \%$ lymphocytes), red blood cell count of 12 cells $/ \mathrm{ml}$, glucose $5.00 \mathrm{mmol} / \mathrm{l}(90 \mathrm{mg} / \mathrm{dl})$, protein $0.58 \mathrm{~g} / \mathrm{l}, \mathrm{IgG}$ index 1.72 (normal $0.28-0.66$ ), and three oligoclonal bands not present in serum. Repeat cytology and HSV PCR were negative, and aciclovir was discontinued.

A CT scan and pelvic ultrasound revealed a calcified hypodense lesion in the left adnexa $(6.0 \times 6.1 \times 7.9 \mathrm{~cm})$. Serum tumor markers were negative. Tests for paraneoplastic and voltagegated potassium channel antibodies were negative, but antibodies to $\mathrm{N}$-methyl-D-aspartate receptors (NMDARs) were identified in serum and CSF (Figure 2). Given the rapid deterioration of the patient's condition, a left salpingooophorectomy was performed, revealing an immature teratoma of the ovary (Figure 3 ).

The patient underwent tracheostomy and gastrostomy tube placement, and was started on intravenous methylprednisolone $1 \mathrm{~g} /$ day for 10 days and plasma exchange (5 exchanges in 10 days), followed by intravenous immunoglobulin (IVIg) $0.4 \mathrm{~g} / \mathrm{kg}$ body weight/day for 5 days. As no improvement was noted, a bolus of cyclophosphamide was given $\left(750 \mathrm{mg} / \mathrm{m}^{2}\right)$. The patient continued on a slow steroid taper. One week later, the abnormal movements subsided and the cardiac arrhythmia resolved. She gradually became more alert and was weaned off the ventilator. She was severely deconditioned with minimal antigravity strength in all extremities. Language, cranial nerves and sensory function were intact. She did not remember the preceding 
2 months, but could form new memories. Two months after discharge, follow-up MRI revealed considerable improvement of the FLAIR hyperintensity in the hippocampi (Figure 1B). Her cognitive functions, memory, and psychiatric evaluation were normal. The neurological examination showed only mild generalized weakness; she could walk without assistance and had started chemotherapy.

\section{DISCUSSION OF DIAGNOSIS}

The syndrome of this patient along with the MRI findings, CSF inflammatory features, and history of an 'ovarian cyst' raised suspicions of the presence of a type of paraneoplastic encephalitis that has recently been described in patients with teratoma. ${ }^{1}$ The disorder associates with antibodies to NMDAR and affects young women, who are then often hospitalized for psychiatric care with diagnoses of acute schizophrenia, catatonia, drug abuse, or malingering. ${ }^{2}$ It is not until the patient develops seizures, autonomic instability, dyskinesias or a decreased level of consciousness that an organic illness is considered. Less frequently, patients present with severe short-term memory deficits resembling limbic encephalopathy. ${ }^{3}$

Box 1 outlines the clinical features of this type of paraneoplastic encephalitis, which often evolves as follows: psychiatric or 'limbic' presentation with seizures, decline of consciousness, dysautonomia and movement disorder, and recovery after treatment. ${ }^{2,4,5}$ At times patients might remain with eyes open, mumbling or unresponsive, with increased muscle tone, similar to catatonia. In $25 \%$ of patients, FLAIR or $\mathrm{T}_{2}$ abnormalities in medial temporal or frontobasal regions are observed, sometimes with mild or transient meningeal enhancement as occurred in the present case. ${ }^{2}$

NMDAR antibodies are present in serum and CSF, usually with intrathecal synthesis, and are sometimes only detected in the CSF. The main target epitopes are in the NR1/NR2 heteromers of the NMDAR. The major antigen is NR1/NR2B, which is predominantly expressed in the hippocampus and forebrain, but reactivity with other NR1/NR2 heteromers is frequently observed. ${ }^{2}$ In the present case, the patient had serum and CSF antibodies that reacted with all NR1/NR2 heteromers (NR2A-NR2D).

The associated tumors are mature (dermoid cyst) or immature teratomas of the ovary; recurrent or bilateral tumors can occur. ${ }^{6}$ A CT scan and
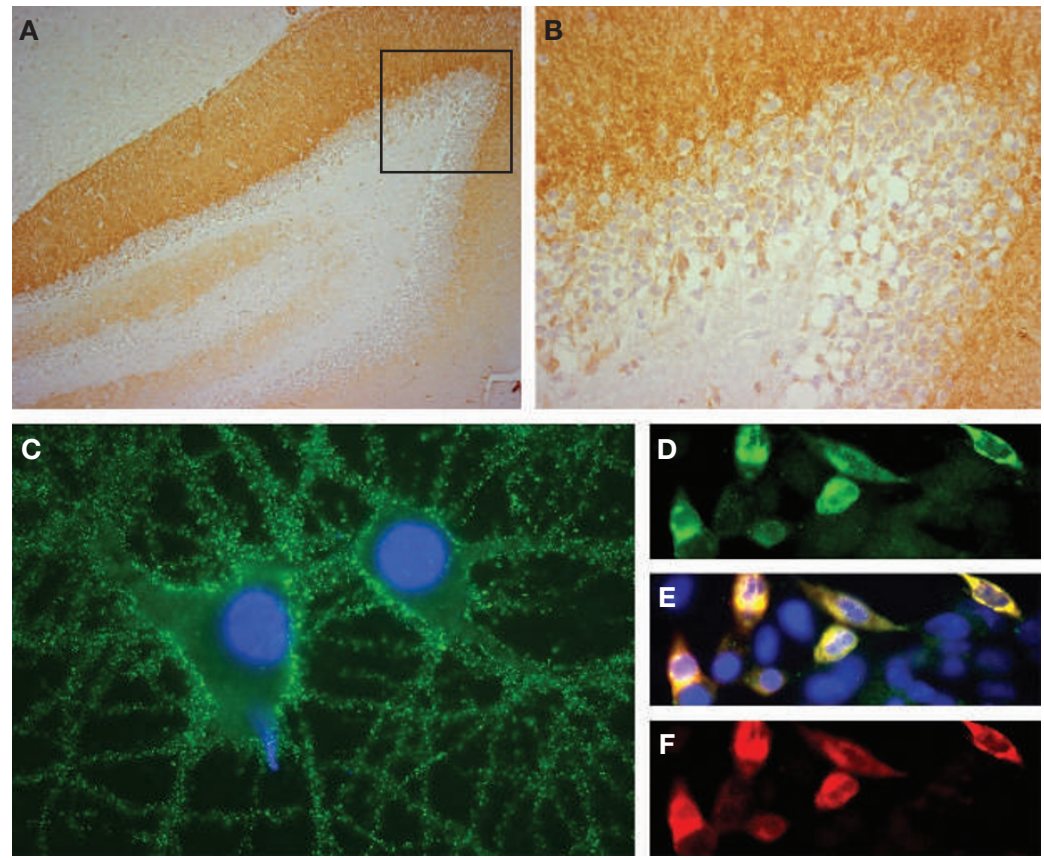

Figure 2 Demonstration of $N$-methyl-D-aspartate receptor antibodies in the patient's cerebrospinal fluid. (A,B) Sagittal section of rat hippocampus incubated with the patient's cerebrospinal fluid (CSF; diluted 1:20). The anti- $N$-methyl-D-aspartate receptor (NMDAR) antibodies produce intense immunolabeling of the molecular layer, adjacent to the granular cells of the dentate gyrus (faintly counterstained with hematoxylin). The area of the dentate gyrus included in the box is shown magnified in panel B. (C) Cultured rat hippocampal neurons incubated with the patient's CSF demonstrate intense immunolabeling of NMDARs contained in the surface of neurons and neuronal processes (nuclei demonstrated by staining with DAPI). (D-F) Confirmation that the CSF antibodies selectively react with NMDARs is shown using human embryonic kidney (HEK293) cells expressing NR1/NR2B heteromers of the NMDAR. The reactivity of the patient's antibodies (green, panel D) co-localize (yellow, panel E) with the reactivity of NR2B-specific antibodies (red, panel F). In addition, the patient's antibodies reacted with NR1/NR2 heteromers containing NR2A, NR2C and NR2D subunits of the NMDAR, which have substantial homology with NR2B (not shown). All immunohistochemical techniques have been reported previously. ${ }^{2}$ Immunoperoxidase method was used in panels $A(100 x)$ and $B(400 x)$, and immunofluorescence in panels $C$ $(800 x)$ and D-F (400x).

ultrasound of the pelvis, or intravaginal ultrasound, usually reveals the tumor. As $70 \%$ of the tumors are benign, PET scans can be negative. ${ }^{1}$ In one patient the teratoma was in the mediastinum. ${ }^{2}$ In all patients studied the NMDAR antibodies reacted with nervous tissue contained in the tumor (J Dalmau, unpublished data). ${ }^{2}$

\section{DIFFERENTIAL DIAGNOSIS}

The current patient presented with low-grade fevers, headaches and anxiety that progressed to paranoia, homicidal ideation, and seizures. These symptoms suggested several toxic and metabolic 

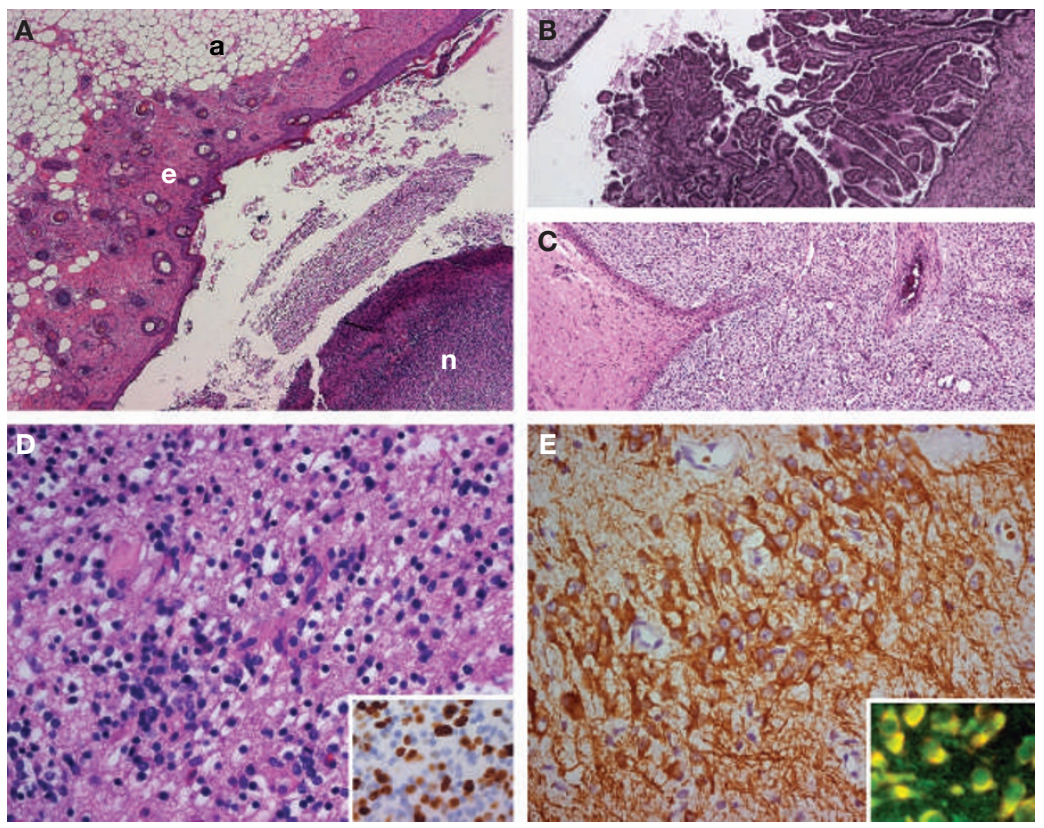

Figure $\mathbf{3}$ Histopathological studies of the patient's teratoma. (A-C) Areas of the tumor containing adipose tissue (a), epithelial tissue (e), and nervous tissue (n), all in the same section (panel A). Other areas contained choroid plexus (panel B), immature mesenchymal tissue (panel C), and bone and cartilage (not shown). These findings are consistent with immature teratoma. (D) Area of the tumor with immature neurons and glial cells. Ki67 immunostaining (inset) shows extensive proliferative activity of the immature glial tissue (30-40\% of the cells). (E) Immunostaining with microtubuleassociated protein 2 (MAP2; a specific marker of neurons and processes) shows an area of immature neurons with an extensive network of neuronal processes. The patient's antibodies reacted with immature nervous tissue expressing $N$-methyl-D-aspartate receptors; the inset shows in yellow the co-localization of the reactivity of patient's antibodies with an antibody specific for the NR2B subunit of the $N$-methyl-D-aspartate receptor.

disorders that were excluded by the patient's history, and by blood and urine testing.

The disorders most frequently considered in the differential diagnosis of 21 patients with anti-NMDAR encephalitis are shown in Table 1 ( $\mathrm{J}$ Dalmau, unpublished data). ${ }^{2}$ In the present case, the CSF inflammatory abnormalities and MRI FLAIR hyperintensity in the patient's temporal lobes were consistent with viral or autoimmune encephalitis. HSV encephalitis was suspected, but several HSV PCRs of CSF were negative, and there was no response to aciclovir. ${ }^{7,8}$ Other viral encephalitides were excluded with appropriate testing.

Most autoimmune encephalitides cause psychiatric symptoms and CSF inflammatory abnormalities; however, MRI scans revealing hyperintensities involving medial temporal lobes are frequent in only two disorders: the
Box 1 Clinical features and diagnostic tests in $\mathrm{N}$-methyl-D-aspartate receptor antibody-associated encephalitis.

Psychiatric symptoms (frequent presentation) Change of personality and behavior, irritability, anxiety, aggressive behavior, delusional thoughts, paranoia, catatonia

Short-term memory loss (rare presentation) Similar to classical limbic encephalitis

\section{Seizures}

Partial complex or generalized seizures

\section{Autonomic instability}

Hyperthermia (sometimes alternating with hypothermia), hypoventilation, fluctuations of blood pressure, tachycardia, bradycardia, constipation, ileus

\section{Abnormal movements (usually without electroencephalogram evidence of epileptic activity)}

Orofacial dyskinesias, dystonic posturing of the extremities, choreoathetoid movements, oculogyric crises, myoclonus, opisthotonos

\section{Cerebrospinal fluid}

Pleocytosis, increased protein concentration, normal glucose, oligoclonal bands and high lgG index

\section{Electroencephalogram}

Frequently: focal or diffuse slow activity during episodes of dyskinesias or abnormal movements Sometimes: epileptic activity

\section{MRI}

Frequently: small areas of FLAIR abnormalities in cerebral cortex (outside the medial temporal lobes), sometimes involving cerebellum and brainstem; transient enhancement of overlying meninges Sometimes: normal or medial temporal and frontal basal FLAIR or $\mathrm{T}_{2}$ abnormalities, with or without involvement of other areas

Abbreviation: FLAIR, fluid-attenuated inversion recovery.

paraneoplastic limbic encephalitides, ${ }^{9}$ and encephalitis associated with voltage-gated potassium channel antibodies. ${ }^{10}$ These disorders (Table 1) are usually associated with characteristic antibodies that were not present in the current patient.

Antibodies to NR2 subunits of the NMDAR have been reported in several other disorders. ${ }^{11,12}$ These antibodies (IgG or IgM) target linear epitopes (detectable by immunoblot), often in the intracellular domain. By contrast, the IgG antibodies of patients with paraneoplastic 
Table 1 Disorders frequently considered in the differential diagnosis of $\mathrm{N}$-methyl-D-aspartate receptor antibody-associated encephalitis. $^{\text {a }}$

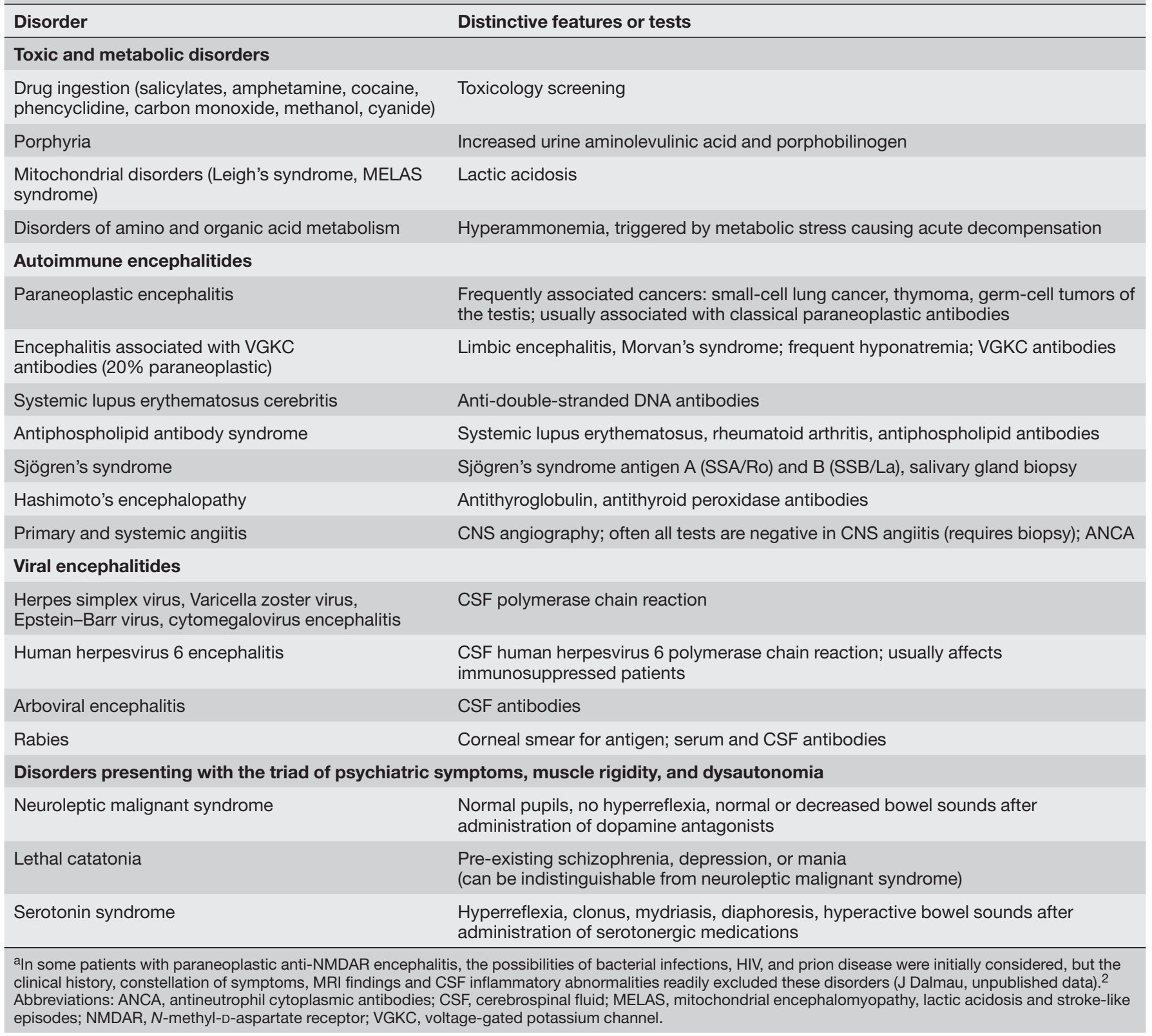

anti-NMDAR encephalitis target conformal extracellular epitopes of NR1/NR2 heteromers (not detectable by immunoblot), and are likely to be pathogenic.

The triad of alteration in mental status, rigidity, and dysautonomia can occur in the neuroleptic malignant syndrome (NMS), serotonin syndrome, or lethal catatonia. Although mechanistically distinct, these disorders can all include muscle hyperactivity, hyperthermia, metabolic acidosis, rhabdomyolysis, elevated creatine kinase (CK), seizures and renal failure. ${ }^{13}$ NMS and serotonin syndrome can be confused with antiNMDAR encephalitis as a result of the fact that most patients with the latter are initially seen in psychiatric centers and might receive neuroleptics or antidepressants. ${ }^{14}$ Once hospitalized, the present patient was not given serotonergic medications and her serum muscle enzymes were normal. Moderate elevation of CK occurred in 1 of 21 patients with paraneoplastic anti-NMDAR encephalitis (J Dalmau, unpublished data). Most 
Acknowledgments We thank Dr Myrna R Rosenfeld for critical review of the manuscript. This work was supported in part by RO1CA107192 (JD) and NS45986 (DL) from the National Institute of Health $(\mathrm{NIH})$.

\section{Competing Interests} The authors declared they have no competing interests. of these patients, however, had inflammatory CSF findings not found in NMS, serotonin syndrome, or lethal catatonia. One patient with paraneoplastic catatonia and ovarian teratoma had elevated CK levels; she partially improved with electroconvulsive therapy, but full recovery was only attained after tumor removal (serum and CSF were not available for antibody analysis). ${ }^{15}$

\section{TREATMENT AND MANAGEMENT}

A study of 12 patients indicated that the optimal management of anti-NMDAR encephalitis is tumor resection and immunotherapy. ${ }^{2}$ Patients with this disorder, however, usually require ventilatory support and intensive care for seizures and autonomic instability, which can delay tumor removal. Treatment with methylprednisolone, plasma exchange or IVIg might result in partial neurological improvement or stabilization allowing resection, although the tumor could grow during the delay. ${ }^{3}$ In some patients, tumor removal results in noticeable neurological improvement in a matter of days or after several weeks. The present patient was diagnosed the day after admission and the tumor was resected that evening. Despite this rapid intervention, she did not start to improve until 3 weeks later, after plasma exchange, IVIg, and a bolus of cyclophosphamide. The latter was used because of previous experience with other patients who did not improve within 1 week of completing plasma exchange or IVIg. Once improvement is noted, most patients continue to improve over weeks or months until full recovery. During improvement, no maintenance immunotherapy is needed. As a result of general deconditioning, and sometimes spinal cord involvement, patients often need prolonged physical therapy. ${ }^{2,16}$

\section{CONCLUSIONS}

Paraneoplastic anti-NMDA receptor encephalitis is potentially lethal, but usually reversible if promptly recognized and treated. It should be suspected in young women with prominent psychiatric symptoms accompanied by seizures, autonomic instability, hypoventilation and dyskinesias. Less frequently it presents as classical limbic encephalitis. The CSF usually shows inflammatory abnormalities, and the MRI can be normal, show transient cerebral cortical or cerebellar abnormalities, or show typical medial temporal lobe hyperintensities. The associated tumors are mature or immature teratomas, usually located in the ovary. Preliminary experience suggests that tumor resection, immunomodulation, intensive care, and physical therapy constitute the best treatment approach.

\section{References}

1 Vitaliani R et al. (2005) Paraneoplastic encephalitis, psychiatric symptoms, and hypoventilation in ovarian teratoma. Ann Neurol 58: 594-604

2 Dalmau J et al. (2007) Paraneoplastic anti-N-methylD-aspartate receptor encephalitis associated with ovarian teratoma. Ann Neurol 61: 25-36

3 Shimazaki H et al. (2007) Reversible limbic encephalitis with antibodies against membranes of neurons of hippocampus. J Neurol Neurosurg Psychiatry 78: 324-325

4 Stein-Wexler R et al. (2005) Paraneoplastic limbic encephalitis in a teenage girl with an immature ovarian teratoma. Pediatr Radiol 35: 694-697

5 Koide R et al. (2007) EFA6A-like antibodies in paraneoplastic encephalitis associated with immature ovarian teratoma: a case report. J Neurooncol 81: 71-74

6 Muni RH et al. (2004) Bilateral horizontal gaze palsy in presumed paraneoplastic brainstem encephalitis associated with a benign ovarian teratoma. J Neuroophthalmol 24: 114-118

7 Tyler KL (2004) Update on herpes simplex encephalitis. Rev Neurol Dis 1: 169-178

8 Wainwright MS et al. (2001) Human herpesvirus 6 limbic encephalitis after stem cell transplantation. Ann Neurol 50: 612-619

9 Gultekin SH et al. (2000) Paraneoplastic limbic encephalitis: neurological symptoms, immunological findings and tumour association in 50 patients. Brain 123: $1481-1494$

10 Vincent A et al. (2004) Potassium channel antibody-associated encephalopathy: a potentially immunotherapy-responsive form of limbic encephalitis. Brain 127: 701-712

11 Mochizuki Y et al. (2006) Acute limbic encephalitis: a new entity? Neurosci Lett 394: 5-8

12 Takahashi Y et al. (2005) Autoantibodies and cellmediated autoimmunity to NMDA-type GluRepsilon2 in patients with Rasmussen's encephalitis and chronic progressive epilepsia partialis continua. Epilepsia 46 (Suppl 5): S152-S158

13 Carbone JR (2000) The neuroleptic malignant and serotonin syndromes. Emerg Med Clin North Am 18: 317-325

14 Boyer EW and Shannon M (2005) The serotonin syndrome. N Engl J Med 352: 1112-1120

15 Lee A et al. (2006) Electroconvulsive therapy in a pediatric patient with malignant catatonia and paraneoplastic limbic encephalitis. J ECT 22: 267-270

16 Taylor RB et al. (1999) Reversible paraneoplastic encephalomyelitis associated with a benign ovarian teratoma. Can J Neurol Sci 26: 317-320 